\title{
Using DevContainers to Standardize Student Development Environments: An Experience Report
}

\author{
Sander Valstar, William G. Griswold, and Leo Porter \\ University of California, San Diego \\ \{avalstar,wgg,leporter\}@eng.ucsd.edu
}

\begin{abstract}
In computer science classes it can be a challenge to ensure every student has a functioning development environment. Running preconfigured servers that provide students with remote access can help mitigate most of these setup issues, however they can also introduce new limitations of their own. We propose using DevContainers to overcome the local machine setup difficulties for the students. DevContainers allow the instructional staff to provision a development environment (a Docker image) with all the correct software versions pre-configured. This development environment can be used on any major OS through Docker. Moreover, through this DevContainer configuration, Microsoft Visual Studio Code can integrate seamlessly with the Docker container to provide an experience for the user that is practically the same as working on the native OS. This work examines the value of employing a DevContainer setup in an Advanced Data Structures course and provides details for those interested in using DevContainers in their courses.
\end{abstract}

\section{CCS CONCEPTS}

-Social and professional topics $\rightarrow$ Computing education; Computer science education; Software engineering education.

\section{KEYWORDS}

Docker, DevContainer, Student development environments

\section{ACM Reference Format:}

Sander Valstar, William G. Griswold, and Leo Porter. 2020. Using DevContainers to Standardize Student Development Environments: An Experience Report. In Proceedings of the 2020 ACM Conference on Innovation and Technology in Computer Science Education (ITiCSE '20), June 1519, 2020, Trondheim, Norway. ACM, New York, NY, USA, 7 pages. https: //doi.org/10.1145/3341525.3387424

\section{INTRODUCTION}

In computer science classes, it can be a challenge to make sure every student has a working development environment. This is especially true when students use their own machines that often have different Operating Systems (OSs) and different, potentially

Permission to make digital or hard copies of all or part of this work for personal or classroom use is granted without fee provided that copies are not made or distributed for profit or commercial advantage and that copies bear this notice and the full citation on the first page. Copyrights for components of this work owned by others than ACM must be honored. Abstracting with credit is permitted. To copy otherwise, or republish, to post on servers or to redistribute to lists, requires prior specific permission and/or a fee. Request permissions from permissions@acm.org.

ITiCSE '20, June 15-19, 2020, Trondheim, Norway

(c) 2020 Association for Computing Machinery.

ACM ISBN 978-1-4503-6874-2/20/06 . \$ \$15.00

https://doi.org/10.1145/3341525.3387424 incompatible, versions of the tools used in the classroom. At our institution, we have historically tried to mitigate this issue by providing pre-configured user accounts on servers that can be accessed on campus in computer labs as well as remotely through ssh. Although this approach alleviates the challenges of setting up a working development environment it also introduces several issues

To create the appropriate server environment, instructors are required to collaborate with a third party on campus that is responsible for installing the necessary software. Because the third party manages many machines for multiple courses, the process can be slow at times and may even delay student access at the start of the term. To avoid this delay, instructors have become reluctant to ask for new software or software updates. As a result, we discovered that the software versions on these lab machines were roughly five years out of date. Unfortunately, this reluctance to update software versions and install new software makes it also harder to innovate and improve the course.

For students, using these pre-configured machines requires that students either be on campus or connect online to work on their assignments. Those connecting remotely may encounter issues while using ssh. For example the connection may not always be stable and some students prefer to work with a GUI which is more tedious to setup over ssh (often resulting in difficulties for students). Finally, students need to use login credentials associated with a campus provided account in order to access the machines and obtaining these credentials can also lead to struggles for students. As a result of these issues, we found in our classes that a portion of students will still attempt to use their own laptops for the programming assignments anyway, often causing another set of difficulties for students and instructional staff.

To overcome these difficulties, we propose using DevContainers [1]. As DevContainers use Docker [2], they allow instructors to provision a development environment (a Docker image) with all the appropriate software versions installed and properly configured. This development environment can be employed on any major OS through Docker. Moreover, we demonstrate how the DevContainer setup allows Microsoft Visual Studio Code (VSCode) [12]-a free text editor with IDE capabilities-to optimally integrate with these Docker environments. From our personal experience as users, we found they integrate so seamlessly that it feels almost the same as working on the native OS.

We studied 416 students in an Advanced Data Structures course at our institution. Course projects are written in $\mathrm{C}++$, which is notorious for having different compilers for different operating systems that all have slightly different standards. Moreover, $\mathrm{C}++$ tools such as GDB and Valgrind can be hard to install on certain OSs $[3,11]$. Hence the course is an excellent candidate for this study. The first author of the paper was a teaching assistant for the course 
and did all of the work to setup the DevContainer configurations for the first programming assignment of the course. We announced that students had the option of either using the lab machines or using the DevContainer before the first programming assignment was released. We surveyed the students at the end of the term on which of the two options they ended up using, why they chose to use this method, if they tried the DevContainer at all, and what their experience had been with the DevContainer. The aim of this experience report is to evaluate how well students value this development environment while providing sufficient details for faculty to adopt our approach in their courses.

\section{THE PROBLEM}

Why is it hard for our students to configure their own machines?

\subsection{Issues of Scale}

Since the majority of our students are majoring in CS, we often expect them to be able to figure out how to install the proper software on their machine. However, many of our CS courses are taken by $400+$ students at the same time and enrollment numbers are still rising [16], so we want to minimize as many avoidable issues as we can. If we ask students to do anything, such as installing a piece of software, there will be at least a couple dozen students who run into problems and addressing those problems can drain teaching resources. As such, we want to minimize the amount of configuring required by students.

\subsection{It Works on My Machine}

In software development, using different versions of tools leads to unexpected issues. For example, the result of using different tools may be that a piece of software works on the machine of the developer, but not on the machine of their colleague or their customer. Such issues are popularly referred to as "it works on my machine"issues. They are somewhat common in software development and can cause serious complications and unexpected extra work when deploying a piece of software to the customer. "It works on my machine"-problems generally occur due to differences in OS, file paths, available libraries, etc.

To help minimize these issues in large classes for the course studied in this paper, instructors require students to work with the exact same tools and versions as the auto grading system. Since the auto grader is running on Linux, it uses tools that were developed for Linux such as GCC, GDB, and Valgrind. This is problematic as our students primarily own Windows or Mac machines (44\% and $46 \%$ of students respectively for the course we studied for this paper) and these Linux tools can be difficult to install on Windows and Mac. Having the students with Windows machines use the Microsoft Visual C++ (MSVC) compiler instead of GCC is not an option as they do not adhere to the same $\mathrm{C}++$ standard. This could lead to issues where a student's code compiles on their own machine, but not on the auto grader, i.e. an "it works on my machine"-issue.

\subsection{Pre-Configured Linux Servers}

We currently mitigate these problems by offering student accounts on Linux machines hosted on campus. These accounts come preconfigured with all the software used in the course. In other words, the students will be able to use the correct software without any need for handling installation or configuration issues. Although this solution has many positives, it has numerous problems as well.

The first issue is that the Linux machines are hosted and maintained by a third party on campus so we lack full administrative control. This means that at the start of every term we have to contact this third party and ask them to configure the machines for our course. Moreover, whenever we want to make a change to the configuration we have to request the change from the third party, leading to lengthy conversations to clarify needs and expectations. For example, in the course studied here, the process of re-configuring the servers took two weeks to complete. As a result of this involved process, instructors generally prefer telling the third party to maintain the configuration used for a previous term of the course. While this mostly works, it results in a situation where the software on the servers rarely gets updated. In addition to having students use out-of-date tools, instructors may become hesitant to make innovations in their course. For instance, instructors have reported hesitancy to make changes to the course because they felt the four week winter break was insufficient to properly setup the systems.

The second issue is with regard to the two ways in which students can access these systems. The first way is by physically going to computer rooms on campus and the second is by connecting remotely over ssh. Thus, students can only work on their programming assignments using the Linux servers if they are either on campus or online.

The third issue is that students encounter problems while working remotely. For example, the connection is not always stable, especially when hundreds of students are working on the same set of servers minutes before the deadline of an assignment. Moreover, some students may prefer using a Graphical User Interface (GUI), which can be set up by using an editor that can connect to a remote server over ssh or by setting up a GUI connection over ssh using X11 forwarding, however this is somewhat tedious to setup so students invariably ask for help configuring this on their machines.

The fourth issue is that students have to be able to log on to the machines using their student credentials. Every term some students are unable to log on to the machines. For example, occasionally students are on the class waitlist for multiple weeks (unable to get access) or are exchange students so their accounts work slightly different than regular students, etc.

\section{WHAT IS A DEVCONTAINER?}

In this section, we detail how a DevContainer works and how one can use it in a course.

\subsection{Docker}

Docker is a way to "run your application anywhere" and it is a fundamental part of how DevContainers work [2]. Docker originally became popular as a way to make deploying software to web servers easier, accelerated by the popularization of microservices and DevOps $[13,15]$. However, that is not its only use. One of the main problems Docker is trying to combat is the "it works on my machine"-issues that occur frequently in software development 


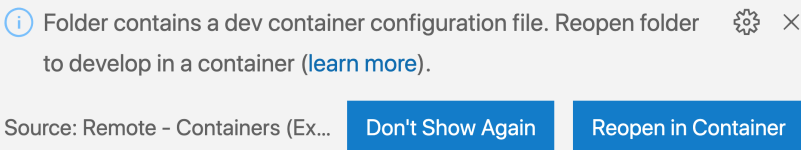

Figure 1: VSCode prompting the user with the option to reopen the project in a DevContainer.

and, as described in Section 2.2, this is exactly one of the issues present in our courses. DevContainers then, seem to be a good fit for our use case.

3.1.1 Docker or Virtual Machines. Virtual machines are another way of addressing this issue [14, 17]. However, virtual machines are slow to start up and take up a great deal of resources from the host system. Moreover, each virtual machine has its own fully loaded operating system. Docker on the other hand is built on a technology called "containerization". Containers share their kernel with the host system which makes them more resource efficient and faster than virtual machines. However, to be able to run, for instance, a Linux container on a Windows machine there is still some level of virtualization required. Currently Docker solves this problem by running a very small Linux virtual machine on Windows. The Linux containers can then share this virtual machine. It is less efficient than running fully without virtualization, but still more efficient than creating a new virtual machine for each container. Moreover, in the future this small virtual machine may no longer be necessary through technologies such as LCOW with Hyper$\mathrm{V}$ isolation (an experimental feature at the time of writing) [5]. Efficiency is important to us as some students may not have the financial resources to buy a powerful machine for their school work.

In summary, Docker containers are an alternative to virtual machines that are more resource efficient. This leads to several benefits, for instance one can easily run a large number of containers on a single machine without exhausting its resources. Moreover, containers start up considerably faster than a virtual machine.

\subsection{Visual Studio Code}

Visual Studio Code (VSCode), not to be confused with the proprietary Visual Studio, is a free and open source text editor with IDE capabilities developed by Microsoft [12]. VSCode runs on all major OSs and it leverages Docker to provide the user with the option of using a DevContainer. If a DevContainer configuration is present in a project VSCode will prompt the user to reopen the project in a DevContainer with a single mouse click (see Figure 1).

\subsection{DevContainer Configuration}

A DevContainer can be configured by creating a hidden directory in a VSCode project. The directory is called ".devcontainer" and contains a json configuration file, a Dockerfile and optionally a docker-compose file for more complex use cases (see Figure 2). Since the devcontainer directory is located in the project it can easily be checked into version control together with the rest of the project. Thus, a new developer (or a student) who checks out the code will immediately have access to the DevContainer.

In the json file the user can specify the name of the DevContainer, which VSCode extensions should be installed, and which directory

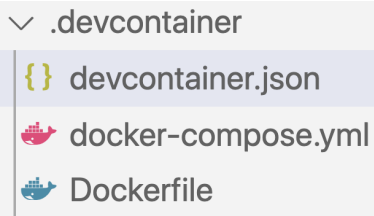

Figure 2: The DevContainer is configured in the .devcontainer directory in the root directory of the VSCode project.

the project should be mapped to in the Docker container. The Dockerfile allows the user to specify a base image using the FROM keyword, usually an OS base image such as "ubuntu:18.04". The RUN keyword can be used to run any commands needed to initialize the container. Usually these are commands that install packages using package manager tools such as apt-get or yum. When the user clicks "Reopen in Container", the Dockerfile and the json file will be used to run a Docker container with all the required software installed. VSCode will automatically set up a connection to the container once it has been created. Microsoft provides pre-configured base projects for a number of programming languages [6].

\section{SETTING UP A DEVCONTAINER FOR A PROGRAMMING ASSIGNMENT}

While using a DevContainer does away with many of the setup issues for students, there is still some setup required from both the instructional team and the students.

\subsection{Setup Work by the Instructional Team}

The instructional team will have to configure a programming assignment base project with a DevContainer configured in it. This DevContainer should have all the required software installed. An example of the setup we used can be found here:

https://github.com/sandervalstar/iticse_devcontainer_demo

\subsection{Setup Work by the Students}

Our students only had to install Docker and VSCode for the $\mathrm{C}++$ development in our class, as opposed to having to install GCC, GDB, Valgrind, and several other tools which can be a real challenge to install on Windows and Mac. Once the project has been opened in the DevContainer (a single mouse click, see Figure 1) the student has access to all of the required tools to complete the assignment. With this setup, students no longer have to be on campus or online while working on the programming assignments. Moreover, they only have to setup two pieces of software (Docker and VSCode) that are popular and should be easy to install on all major OSes. One caveat to installing Docker is that it requires Hyper- $\mathrm{V}$ on Windows which is not supported by Windows Home-edition. However, students can resolve this by upgrading for free to Windows Education-edition.

\section{STUDY DESIGN}

We studied 416 students in an upper-division Advanced Data Structures course with a total enrollment of 449 students divided over 3 sections and 2 different instructors. The course had 2 graduate student teaching assistants, 11 undergraduate student head tutors and 26 undergraduate student tutors. Our study was conducted at a 
large public research-focused institution in North America and has institutional human subjects approval. The upper division course we studied has four programming assignments which have to be completed in $\mathrm{C}++$. As a part of our study, we allowed students the choice between working on the Linux servers or in a DevContainer.

\subsection{Research Questions}

We posed the following Research Questions (RQs):

(1) Do the majority of students prefer using the DevContainer or the Linux servers, and why?

(2) Are students from underrepresented groups more or less likely than other students to prefer using the DevContainer?

(3) What advantages and disadvantages do students experience with the DevContainer compared to the Linux servers?

(4) What is the cost of the DevContainer for the instructional team (e.g., will we have to help more students than usual)?

(5) What does the instructional team think of the DevContainers?

\subsection{Data Collection}

During the first week of the term we collected demographic data about our students for RQ 2. In the last programming assignment, we included a final survey on the topic rewarded with participation credit. The questions on this survey all targeted RQ's 1, 3, and 4 . For RQ 4 we also collected data from the class' Piazza board. Piazza is an online class forum where students can post questions [10]. For RQ 5 we administered a voluntary anonymous survey to the instructional team which was filled out by 11 out of 41 members (all were undergraduate tutors or head tutors).

\section{RESULTS}

According to our end-of-term survey, $71 \%$ of students developed in the DevContainer while 29\% of students developed on the Linux server. The students overwhelmingly recommended students in following terms to use the DevContainer with $70 \%$ of students recommending or strongly recommending it and only a mere $8 \%$ of students recommending against using it . The Linux server was recommended by $28 \%$ of students whereas $34 \%$ of students would not recommend or strongly not recommend students in following terms to use the Linux server.

\subsection{Reasons for Student Preferences}

We asked students an open-ended question for the reason why they used the DevContainer instead of the Linux server or vice versa. For both questions we cleaned up the responses using Python's Natural Language Tool Kit (NLTK) [7]. The data cleaning included filtering out stop words and using a word lemmatizer to group similar words under the same term. We then performed a word collocation analysis on the responses to see which were the most common reasons for their respective preference.

6.1.1 Reasons For Preferring the DevContainer. We identified the best commonly collocated trigrams, scored by likelihood ratio and grouped all common trigrams that pertained to the same reason under a single sentence with the group size in parentheses at the end. In case a trigram did not fully make sense by itself we did a manual search for the keywords to understand the most common meaning of the trigram. Clarifications are provided in square brackets.

(1) I like the VSCode editor. (7)

(2) Working on my local machine is more convenient than sshing on to the Linux server. (5)

(3) I wanted to try something new. (2)

(4) '[Does not require] internet', 'connection', 'work'

(5) 'want', 'freedom', 'able [to work from home/anywhere]'

From these results we can conclude that a major reason why the students preferred the DevContainer was that they believed that the DevContainer was what allowed them to use the VSCode editor. Actually, the students could have connected to the Linux server using VSCode's ssh functionality allowing them to use VSCode with the Linux server, but many students may not have been aware of this option. Other reasons include wanting to try something new and some students mentioned that they would not be able to use the Linux server after they had graduated, but they would still be able to use the DevContainer. Students also preferred working on their local machine over connecting to the Linux server. Some students mention that having a bad WiFi connection has a massive impact on the user experience of using the Linux server and they dislike being dependent on good WiFi because the WiFi performance on campus is unreliable as one student described wittily: "[School]'s wifi runs slower than I do".

6.1.2 Reasons For Preferring the Linux Server. We performed a similar analysis for students who preferred working on the Linux server.

(1) I am accustomed to the Linux server and the Vim editor as we used it in previous classes. (4)

(2) It took too much time to get the DevContainer to work. (3)

(3) The DevContainer constantly gives a popup saying "reconnecting" and VSCode freezes until it disappears. (2)

(4) 'laptop', 'could [not]', 'handle [running the DevContainer ]'

Based on these results we believe that students who prefer to use the Linux server primarily do so because they gained familiarity with the Linux server in previous courses. Since we are studying an upper-division course, it is not surprising the students had grown accustomed to using the Linux server and Vim because they have been using this setup in nearly all courses with programming components leading up to this course. Other reasons are that these students had trouble figuring out how to get the DevContainer to work, they experienced a "reconnecting" issue with the DevContainer, or their laptop may not have been powerful enough to run the DevContainer.

\subsection{Preferences of Underrepresented Groups}

When introducing new approaches into CS classrooms, it is important to verify that we are not unintentionally making the barrier to study CS higher than it already is for underrepresented groups. The necessary demographic information was obtained using a voluntary survey during the first week of class. We studied a total of 202 students for which we had both the demographic information and their preference for the DevContainer or the Linux server. The gender of respondents was $68 \%$ men, $29 \%$ women, $3 \%$ nonbinary and the responses to race/ethnicity were $64 \%$ Asian, $19 \%$ 
Caucasian (non Hispanic/Latinx), 9\% Hispanic/Latinx, 0\% Black, $7 \%$ other races/ethnicities. We were interested to see whether or not students from underrepresented groups had a stronger preference for DevContainers or the Linux server compared to the other students. However, almost all groups we analyzed had a more than $60 \%$ preference for the DevContainer. Interestingly, people who identified as Asian had the highest preference for the DevContainer with $75 \%$. Students who identified as other races/ethnicities were the only group with a slight preference for the Linux server at $53 \%$. Unfortunately, some of our underrepresented groups are quite small, making it hard to attach statistical significance to these findings. Indeed after running chi-squared tests at a significance level of 0.05 , we found no significant correlation between gender or race/ethnicity and a stronger preference for the DevContainer or the Linux server.

\subsection{Disadvantages of the DevContainer and the Linux Server}

6.3.1 Disadvantages of the DevContainer. We asked all students to name at least one disadvantage of the DevContainer compared to the Linux server. These were the most commonly collocated trigrams.

(1) The DevContainer can give issues with large files such as big data sets (2)

(2) 'take', 'lot', 'storage'

(3) 'take', 'much', 'memory'

(4) 'run', 'significantly', 'slower'

(5) 'take', 'time', 'get [to work]'

A disadvantage of the DevContainer seems to be that it does not handle big data sets well, possibly this issue occurs when a too conservative amount of resources is allocated to Docker. Furthermore, a Docker image may consume considerable storage on disk. On the other hand, in our experience, the Linux sever was notorious for students exceeding their disk space quota. Students reported the DevContainer also consumes a great deal of working memory on their laptop and may run slower than the Linux server, depending on the speed of the student's machine. Moreover, Docker takes some time to set up the first time.

6.3.2 Disadvantages of the Linux Server. We also asked all students to name at least one disadvantage of the Linux server compared to the DevContainer and found the following commonly collocated trigrams.

(1) Need to use Vim instead of VSCode. (6)

(2) Need to log in every time over ssh. (4)

(3) Need a stable internet connection. (3)

(4) Less user friendly. (3)

(5) '[Need to go to campus to] use', 'lab', 'computers'

As in Section 6.1.1, students' preference for VSCode over Vim clearly appears again, despite previous experience with Vim. Furthermore, students disliked having to log in every time through ssh before being able to do work. Students also disliked the need for an internet connection (or being on campus) to be able to work on the Linux server. As mentioned before, internet speed had a heavy impact on the user experience.
How difficult/easy was it to set up the DevContainer?

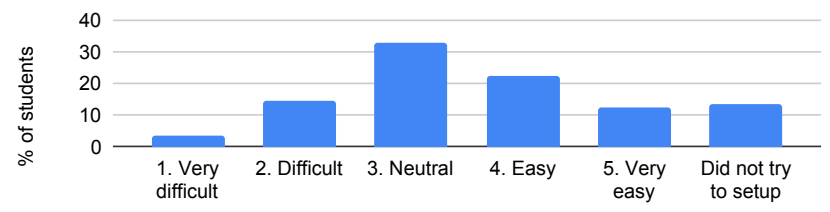

Figure 3: A large percentage of students was neutral or leaned towards easy when asked about whether the DevContainer was easy to set up.

\section{I had to request help for setting up my DevContainer}

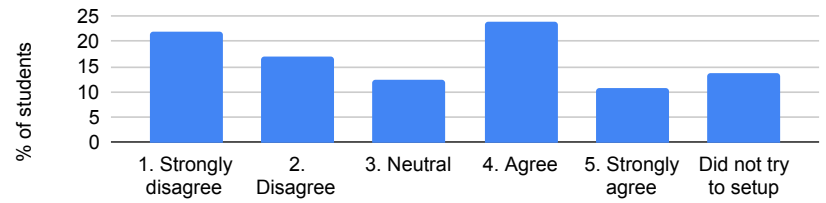

Figure 4: Students were divided on whether or not they needed help from an instructor or classmate with setting up the devcontainer, with $35 \%$ of students needing help and $39 \%$ not needing help.

\subsection{Cost to The Instructional Team}

To examine the impact using DevContainers may have on the workload of our students and instructional team, we analyzed the endof-term student survey, the Piazza forums, the instructional team Slack channel, and the instructional team survey.

6.4.1 End-of-Term Survey. On the survey, 18\% of students reported that the DevContainer was difficult or very difficult to set up whereas 35\% of students reported that the DevContainer was easy or very easy to set up (see Figure 3). The remaining students were neutral or did not try the DevContainer.

We also asked students whether they needed any help while setting up the DevContainer, to which 35\% responded they needed help or strongly needed help and 39\% did not need help (see Figure 4).

Students seemed to require less help for setting up their Linux server account with $52 \%$ of students not needing any help and only $17 \%$ reporting to have needed help (see Figure 5). However, this may be explained by the fact that we study an Advanced Data Structures course with multiple prerequisite programming courses, thus most students have used the Linux server before in several other courses. It is therefore not all that surprising that they no longer require help with figuring out the server. It is perhaps more surprising that $17 \%$ of students still needed help.

6.4.2 Piazza. Our Piazza forum board contained over 3,000 posts by the end of the term. We wrote a Selenium [8] web crawler that downloaded all the posts and then wrote a script to count the number of posts which mentioned the DevContainer, Docker, or issues related to the DevContainer. This resulted in a total of 98 posts. We did the same for the Linux server and found a total of 108 posts. Given that, as mentioned at the start of Section 6, there were considerably more students using the DevContainer these results show less traffic for the DevContainer. 
I had to request help for setting up my Linux sever account

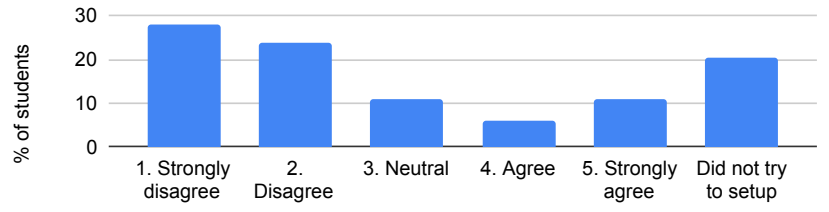

Figure 5: Students seemed to require less help for setting up their Linux server account. This may be explained by the fact that these are all upper division students who have mostly used the Linux server before in multiple prior courses.

6.4.3 Slack. Our instructional team's Slack channel contained over 12,000 messages in total at the end of the term. We downloaded our messages in json format from the Slack website and analyzed them in a similar fashion for the same topics as we did for the Piazza posts. We counted a total of 120 messages related to the DevContainer and 147 messages related to the Linux server.

6.4.4 Instructional Team Survey. The voluntary survey we released to the instructional team contained the following question: "I had to help many students with setting up their DevContainer" for which $64 \%$ responded agree or strongly agree and $18 \%$ responded disagree or strongly disagree. For the Linux server we asked a similar question to which $9 \%$ responded agree and $64 \%$ responded disagree. From the instructional team's point of view then, students required more in-person assistance with the DevContainer.

\subsection{Instructor Opinions}

On the survey, the instructional team overwhelmingly recommended instructors in future terms to use a DevContainer with no one recommending against using it. Some members believed we should have devoted more attention to helping students set up their DevContainer in the beginning of the term in order to prevent questions.

\section{DISCUSSION}

The majority of our students used and preferred the DevContainer. The most common reasons reported were the editor and being able to work on their local machine. The main reported disadvantages of the DevContainer were that it did not handle big data files well and can be slow depending on the speed of the student's laptop. Both students and instructors report that more help was provided for the DevContainer compared to the Linux server. However, our analysis of Piazza and Slack showed that there is less traffic related to the DevContainer when compared to the Linux server on both platforms, especially when adjusted for student usage.

One could argue that setting up the DevContainer was hard for the students compared to using the Linux server and therefore it is not significantly easier for the students than just installing the required software on their own machine. However, we disagree with that narrative, because we found the most difficult part to setting up the DevContainer is installing Docker. After a student has a working Docker installation, running the DevContainer is as simple as the click of a single button. Hence, we hypothesise these DevContainer setup difficulties would only occur in the first course where students have to use a DevContainer. As such, we believe that if the DevContainer had been adopted throughout our curriculumas the Linux server has been-we would see significantly fewer issues with setup in our upper-division course. In contrast, having students install all the software on their own machine will lead to repeated setup issues as different courses will require different software. For instance, the course we studied is the first course in which students have to use $\mathrm{C}++$ so all the software tools would be new. Moreover, we suspect that in the first course in which students have to use the Linux server at our institution they will need significantly more assistance than they did in our course.

\section{THREATS TO VALIDITY}

We find that a major reason for the students preferring the DevContainer was that they preferred using VSCode. We did not expect this since the students have been using Vim for most programming courses since their first year and should be more than comfortable using it at this point. Moreover, students could actually use VSCode to connect to the Linux server using VSCode's ssh function, but many students may not have been aware of this capability. However, despite this threat to some of our findings, our study also clearly uncovered that many students have a preference for a system that allows them to work offline on their own machine. A final threat to validity is that the first author was also a teaching assistant for the course and may have imparted his enthusiasm for DevContainers to the students or course staff.

\section{CONCLUSION}

In this study, we found that the majority of students used our DevContainer setup and generally viewed it favorably-recommending faculty use it in other courses. From the instructor's point of view there are several advantages. For instance, there is no need to communicate with the maintainers of the Linux server to configuring the machines. This offers more control to the instructor about the development environment the students use, which leads to faster turnaround times for improvements to the programming assignments.Another benefit is that the DevContainer's Docker configuration can be used almost without change on auto grading systems such as Gradescope [4]. This ensures that there will be no "it works on my machine"-issues with the auto grader. Furthermore, the DevContainer allows for easy sharing of programming assignments between instructors as the tooling infrastructure is shipped with the assignment. This could be useful for programming assignments sharing platforms such as the SIGCSE symposium's Nifty Assignments [9]. Finally, DevContainers are not a research or hobby project, but a professional tool supported by Microsoft. Hence, they can be expected to continue to be usable and supported in the future. There remain some barriers to using the DevContainer, specifically Docker can be a hurdle to set up for the first time. However, we suspect that this is only an issue in the first course in the curriculum that introduces DevContainers to the students as for following courses students will come into the course with a working Docker configuration. Moreover, we find that despite the initial barrier of setting up the DevContainer the vast majority of students had a clear preference for it over the Linux server. 


\section{REFERENCES}

[1] DevContainers. https://code.visualstudio.com/docs/remote/containers

[2] Docker. https://www.docker.com/

[3] GDB: The GNU Project Debugger. https://www.gnu.org/software/gdb/

[4] Gradescope - an online grading platform. https://www.gradescope.com/

[5] LCOW: Linux Containers on Windows with Hyper-V isolation. https://docs.microsoft.com/en-us/virtualization/windowscontainers/ deploy-containers/linux-containers

[6] Microsoft DevContainer Examples. vscode-remote-try-python

[7] Python Natural Language Tool Kit (NLTK). https://www.nltk.org/
[8] Selenium with Python. https://selenium-python.readthedocs.io/

[9] SIGCSE Nifty Assignments. http://nifty.stanford.edu/

[10] Slack - messaging for work. https://slack.com/

[11] Valgrind - an instrumentation framework for building dynamic analysis tools https://valgrind.org/

[12] Visual Studio Code. https://code.visualstudio.com/
[13] A. Balalaie, A. Heydarnoori, and P. Jamshidi. 2016. Microservices Architecture Enables DevOps: Migration to a Cloud-Native Architecture. IEEE Software 33, 3 (May 2016), 42-52. https://doi.org/10.1109/MS.2016.64

[14] David P. Harvie, Jason R. Cody, Christopher Morrell, and Tanya T. Estes. 2019. Using Virtual Machines to Enhance the Educational Experience in an Introductory Computing Course. In Proceedings of the 20th Annual SIG Conference on Information Technology Education (SIGITE '19). Association for Computing Machinery, New York, NY, USA, 28-32. https://doi.org/10.1145/3349266.3351401

[15] Sam Newman. 2015. Building Microservices (1st ed.). O’Reilly Media, Inc.

[16] Eric Roberts, Tracy Camp, David Culler, Charles Isbell, and Jodi Tims. 2018. Rising CS Enrollments: Meeting the Challenges. In Proceedings of the 49th ACM Technical Symposium on Computer Science Education (SIGCSE '18). ACM, New York, NY, USA, 539-540. https://doi.org/10.1145/3159450.3159628

[17] Geoff Stoker, Todd Arnold, and Paul Maxwell. 2013. Using Virtual Machines to Improve Learning and Save Resources in an Introductory IT Course. In Proceedings of the 14th Annual ACM SIGITE Conference on Information Technology Education (SIGITE '13). Association for Computing Machinery, New York, NY, USA, 91-96. https://doi.org/10.1145/2512276.2512287 